\title{
Groupe de travail sur la vaccinovigilance du Canada
}

\author{
Ahmadipour $\mathbf{N}^{1^{*}}$, Toth $\mathrm{E}^{2}$, Law $\mathrm{BJ}^{1}$ \\ ${ }^{1}$ Centre de l'immunisation et des maladies respiratoires infectieuses, Agence de la santé publique du Canada, Ottawa \\ (Ontario) \\ ${ }^{2}$ Direction de la protection de la santé publique, Ministère de la Santé et des Services sociaux du Québec, Québec (Québec) \\ * Correspondance : Nooshin.Ahmadipour@phac-aspc.gc.ca
}

Résumé

Le Groupe de travail sur la vaccinovigilance a été créé en 2004, dans le cadre de la Stratégie nationale d'immunisation (SNI) visant à renforcer la sécurité des vaccins au Canada. Le Groupe réunit des représentants de tous les programmes d'immunisation à l'échelle fédérale, provinciale et territoriale au pays, ainsi que des organismes de réglementation de Santé Canada et du réseau de surveillance en milieu hospitalier du programme canadien de surveillance active de l'immunisation (IMPACT). Le Groupe de travail sur la vaccinovigilance œuvre en vue d'harmoniser la surveillance de la sécurité des vaccins ainsi que la déclaration et la gestion des événements indésirables à l'échelle canadienne en élaborant et en observant des lignes directrices nationales, et en recherchant les meilleures pratiques en matière de pharmacovigilance, notamment par la formation. Le Groupe fournit également un réseau sentinelle national sur la sécurité des vaccins utilisant plusieurs mécanismes permettant la communication rapide de renseignements au sujet d'enjeux émergents de sécurité, afin de permettre une intervention efficace en matière de santé publique. Les activités du Groupe de travail sur la vaccinovigilance se caractérisent par leur caractère toujours alerte. Le rôle et les activités du Groupe de travail sur la vaccinovigilance devraient contribuer à apaiser les préoccupations au sujet de la sécurité des vaccins à l'origine des réserves à leur sujet, ayant pour effet de diminuer l'efficacité de la vaccination.

\section{Introduction}

La surveillance de la sécurité des vaccins, à l'échelle locale, provinciale, territoriale et nationale a toujours fait partie de l'engagement du Canada à l'égard de la vaccination. En 1999, cet engagement s'est avéré édifiant au vu de la Stratégie nationale d'immunisation (SNI), une initiative pluriannuelle collaborative fédérale-provincialeterritoriale, mise sur pied afin de renforcer la vaccination au Canada (1). La sécurité des vaccins était l'une des cinq composantes principales de la Stratégie. Le rapport de 2003 sur la Stratégie a décrit certaines lacunes et limitations principales du système de sécurité, notamment : le manque de lignes directrices nationales pour la déclaration, la vérification, la gestion et la communication d'informations portant sur les effets secondaires suivant l'immunisation (ESSI), le besoin d'obtenir en temps opportun des données d'échelle nationale sur ces effets secondaires, ainsi qu'une capacité plus solide d'intervenir en cas de problèmes de sécurité urgents liés à la vaccination.

Au chapitre des stratégies visant à améliorer la surveillance de la sécurité des vaccins et l'intervention en santé publique, on compte l'établissement d'un réseau de personnes-ressources spécialisées en matière de sécurité des vaccins à l'échelle fédérale, provinciale et territoriale, et ce, dans toutes les compétences. De multiples rôles ont été envisagés pour le réseau, dont : la surveillance et la déclaration d'ESSI, la détermination de dangers potentiels portant sur la sécurité des vaccins et les solutions à cet égard, la communication, ainsi que la capacité d'intervenir de façon coordonnée en cas d'urgence nationale.

Ce réseau a été créé en 2004 : il s'agit du Groupe de travail sur la vaccinovigilance. Son nom a été choisi avec soin : le mot vigilance vient du latin « vigilare », qui signifie le fait d'être éveillé ou à l'affût (2). La 
pharmacovigilance des vaccins comprend la science et les activités liées à la détection, à l'évaluation, à la compréhension, à la prévention et à la communication des effets secondaires suivant l'immunisation ou de tout autre problème lié à l'immunisation ou aux vaccins (3). Le but du présent document est de décrire la structure, les processus et les extrants du Groupe de travail sur la vaccinovigilance, ainsi que de montrer comment surveiller la sécurité des vaccins au Canada.

\section{Méthode du Groupe de travail sur la vaccinovigilance}

Le Groupe de travail sur la vaccinovigilance œuvre en vue :

- de développer des lignes directrices et des procédures nationales pour la surveillance, la déclaration et la gestion des ESSI;

- de servir de forum national permettant de déterminer, de communiquer et de promouvoir les pratiques exemplaires en matière de sécurité des vaccins, y compris au moyen de formation sur la déclaration et la gestion des ESSI;

- de fournir un réseau sentinelle national sur la sécurité des vaccins pouvant rapidement détecter et intervenir en cas d'enjeux ou de signaux émergents liés à la sécurité des vaccins.

Le groupe de travail comporte deux coprésidents, un à l'échelle fédérale, et l'autre, à l'échelle provinciale. Le groupe est principalement composé de représentants de chaque province et territoire, sauf l'Ontario qui en comporte deux : l'un du ministère de la Santé, qui assure la supervision de la vaccination, et l'autre, de Santé publique Ontario, qui supervise la surveillance des ESSI. Les représentants chargés de la liaison sont des membres non votants provenant des ministères et organisations suivants : des représentants du réseau du Programme canadien de surveillance active de l'immunisation (IMPACT); deux représentants d'organismes de réglementation des vaccins de Santé Canada relevant de la Direction des produits de santé commercialisés (DPSC) et de la Direction des produits biologiques et des thérapies génétiques de Santé Canada, quatre représentants de compétence fédérale assumant des responsabilités liées aux programmes de vaccination, dont la Direction générale de la santé des Premières nations et des Inuits (DGSPNI), le ministère de la Défense nationale (MDN), la Gendarmerie royale du Canada (GRC) et le Service correctionnel du Canada (SCC). Les représentants chargés de la liaison apportent des connaissances et une expertise au groupe de travail, en exprimant leur point de vue et en assurant une rétroaction à leur organisation respective, comme le permet le Groupe de travail sur la vaccinovigilance.

Le Groupe de travail sur la vaccinovigilance relève du Comité canadien sur l'immunisation ( $\mathrm{CCl}$ ), qui a notamment été constitué en raison des recommandations de la Stratégie nationale d'immunisation, dont le mandat est de faciliter la réalisation de la Stratégie. Le CCI relève de son côté de hauts comités faisant partie du Conseil du Réseau pancanadien de santé publique, qui sont eux-mêmes chapeautés par la Conférence des sous-ministres fédéral, provinciaux et territoriaux de la Santé.

\section{Accomplissements du Groupe de travail sur la vaccinovigilance}

En raison des difficultés repérées dans le rapport de 2003 sur la SNI, le Groupe de travail sur la vaccinovigilance a pris plusieurs mesures, dont l'élaboration de procédures et de lignes directrices nationales normalisées, l'établissement d'un outil de communication en ligne d'alertes ESSI et l'augmentation de la surveillance au cours de la pandémie de grippe H1N1 de 2009 (tableau 1). 
Tableau 1 : Activités et accomplissements du Groupe de travail sur la vaccinovigilance

\begin{tabular}{|c|c|c|}
\hline $\begin{array}{l}\text { Difficultés/lacunes liées à la sécurité } \\
\text { des vaccins établies dans le rapport } \\
\text { final de } 2003 \text { sur la SNI }\end{array}$ & Activités clés & Réalisations particulières \\
\hline $\begin{array}{l}\text { Absence de procédures et de lignes } \\
\text { directrices nationales normalisées touchant } \\
\text { les ESSI }\end{array}$ & $\begin{array}{l}\text { Élaboration de lignes } \\
\text { directrices et de } \\
\text { procédures nationales } \\
\text { pour la surveillance et } \\
\text { la gestion des ESSI } \\
\text { afin d'harmoniser les } \\
\text { activités de } \\
\text { surveillance de la } \\
\text { sécurité des vaccins } \\
\text { au Canada. }\end{array}$ & $\begin{array}{l}\text { - } \quad \text { Définitions nationales de cas d'ESSI (4). } \\
\text { Révision du formulaire national de déclaration d'ESSI } \\
\text { pour faciliter la déclaration normalisée d'ESSI, } \\
\text { notamment, par la saisie d'éléments principaux des } \\
\text { définitions de cas d'ESSI. } \\
\text { - } \quad \text { Manuel national de l'utilisateur pour guider la } \\
\text { - } \quad \text { Méclaration d'ESSI (4). } \\
\text { Mise à jour des coordonnées des personnes- } \\
\text { ressources fédérales-provinciales-territoriales sur le } \\
\text { site Web de l'Agence de la santé publique du } \\
\text { Canada pour faciliter la déclaration et la } \\
\text { communication des enjeux liés aux ESSI. } \\
\text { Établissement de procédures normalisées pour } \\
\text { - } \quad \text { Nlusieurs aspects de la déclaration d'ESSI. } \\
\quad \text { Normalisation des abréviations liées à la vaccination. }\end{array}$ \\
\hline $\begin{array}{l}\text { Besoin accru de données d'ESSI en temps } \\
\text { opportun. }\end{array}$ & $\begin{array}{l}\text { Forum national pour } \\
\text { communiquer et } \\
\text { promouvoir les } \\
\text { pratiques exemplaires } \\
\text { en matière de sécurité } \\
\text { des vaccins. }\end{array}$ & $\begin{array}{l}\text { - Création et utilisation d'un outil en ligne d'alertes sur } \\
\text { les ESSI (par l'intermédiaire du Réseau canadien de } \\
\text { renseignements sur la santé publique). } \\
\text { Procédures normalisées de déclaration accélérée } \\
\text { d'ESSI graves et hautement prioritaires. } \\
\text { - Ébauche d'annexe technique sur les ESSI relative } \\
\text { aux ententes fédérales, provinciales et territoriales de } \\
\text { partage de données. }\end{array}$ \\
\hline $\begin{array}{l}\text { Besoin de renforcer la capacité } \\
\text { d'intervention en cas d'urgence. }\end{array}$ & $\begin{array}{l}\text { Réseau sentinelle } \\
\text { national sur la } \\
\text { sécurité. }\end{array}$ & $\begin{array}{l}\text { - Surveillance et partage de données accrus durant la } \\
\text { pandémie de grippe H1N1 de } 2009 \text { et campagnes de } \\
\text { masse subséquentes sur la grippe saisonnière au } \\
\text { moyen de téléconférences et de rapports } \\
\text { hebdomadaires abrégés sur les ESSI. } \\
\text { Ébauche de protocole d'intervention en présence de } \\
\text { signes d'ESSI. }\end{array}$ \\
\hline
\end{tabular}

Par exemple, la surveillance accrue au cours de la pandémie de grippe H1N1 de 2009 a été effectuée au moyen de téléconférences hebdomadaires du Groupe de travail sur la vaccinovigilance ayant permis des communications rapides au sujet de préoccupations émergentes en matière de sécurité des vaccins. Chaque administration fédérale-provinciale-territoriale a participé à ces téléconférences, et a fourni des dénombrements hebdomadaires d'ESSI à l'Agence de la santé publique du Canada (ASPC) comprenant des ESSI graves, ainsi que des ESSI particulièrement préoccupants, comme des chocs anaphylactiques, des convulsions fébriles et le syndrome de Guillain-Barré. Ces efforts ont permis à l'ASPC de fournir des rapports aux intervenants, y compris le public, et ce, presque en temps réel, au sujet de la sécurité du vaccin pandémique pendant et après la campagne de masse.

Les leçons apprises en 2009 ont été appliquées aux campagnes de vaccination de masse subséquentes. Depuis 2010, le Groupe de travail sur la vaccinovigilance a établi des appels hebdomadaires au début de chaque campagne de grippe saisonnière afin de communiquer des données sur la sécurité des vaccins et d'examiner tout enjeu potentiel portant sur la sécurité des vaccins, et ce, en temps opportun.

Les signes potentiels touchant la sécurité des vaccins détectés à l'échelle locale, provinciale, fédérale ou internationale sont communiqués rapidement à tous les membres du Groupe de travail sur la vaccinovigilance à l'aide d'un outil de communication sécuritaire de santé publique, le Réseau canadien de renseignements sur la santé publique. Les enjeux de sécurité des vaccins nécessitant une attention immédiate sont communiqués par téléphone ou par courriel et des conférences téléphoniques urgentes ont lieu au besoin. 
Davantage d'information au sujet du Groupe de travail sur la vaccinovigilance et ses membres est accessible dans son guide de déclaration d'ESSI (4).

\section{Orientations futures}

À l'heure actuelle, le Groupe de travail sur la vaccinovigilance élabore un protocole d'intervention en présence de signes d'ESSI qui décrit les processus et les mesures requises en vue d'une gestion rapide de tout signe de problèmes de sécurité des vaccins émergeant ou nouvellement détecté. Ce protocole décrira les procédures portant notamment sur la détection des signes, la validation, l'analyse et l'établissement de priorités, l'évaluation des signes, les communications et les mesures nécessaires pour chaque ordre du gouvernement, ainsi que les recommandations finales. À chaque étape, les rôles et responsabilités en matière de santé des compétences fédérales-provinciales-territoriales, de l'ASPC et des organismes de réglementation des vaccins de Santé Canada seront clairement décrits, notamment, au moyen d'un plan de communication continue du début à la fin lorsqu'une recommandation est effectuée, ou lorsqu'un signal est considéré comme étant faux. Les annexes du protocole comprendront des modèles généraux et propres aux ESSI, ainsi que des listes de vérification pour faciliter la collecte de données rapide.

\section{Conclusion}

Depuis sa mise sur pied en 2004, le Groupe de travail sur la vaccinovigilance a fourni un forum fédéral-provincialterritorial essentiel à l'intention des travailleurs de première ligne, au sujet de la vaccination et de la vaccinovigilance et a développé une capacité de communication rapide de données sur la sécurité des vaccins. Toutefois, il reste bon nombre de défis à relever. Une priorité essentielle pour la SNI a été de disposer de personnel spécialisé en matière de sécurité des vaccins, à l'échelle locale, provinciale, territoriale et fédérale. À une époque d'austérité et de crises de santé publique inattendues, il est parfois difficile de respecter cette priorité. L'incidence des lacunes a été révélée clairement au cours de la pandémie de 2009. À la suite de cette expérience, la planification en matière de pharmacovigilance en contexte de pandémie s'est concentrée sur des efforts visant à s'assurer que les principales priorités de la SNI pouvaient être mises en œuvre sur une base quotidienne. Des projets pilotes mis en œuvre par le Groupe de travail sur la vaccinovigilance pour combler les autres lacunes sont décrits dans le présent supplément $(5,6)$.

Depuis la création du Groupe de travail sur la vaccinovigilance, de nombreuses préoccupations potentielles en matière de sécurité des vaccins se sont présentées, mais bien peu se sont avérées fondées. Une grande partie des travaux du Groupe de travail sur la vaccinovigilance n'est pas perceptible par le public, les vaccinateurs et autres professionnels de la santé. Toutefois, il est important que ceux-ci soient au courant que la vaccinovigilance constitue une activité quotidienne continue et coordonnée à l'échelle canadienne. Ainsi, l'absence de préoccupation importante en matière de sécurité ne découle pas d'une absence de vigilance et de capacité d'intervenir en cas de problème.

\section{Remerciements}

Des remerciements sont adressés à Wikke Walop, qui, dans le cadre de ses activités en matière de sécurité des vaccins à l'ASPC, a jeté les bases de ce qui allait devenir le Groupe de travail sur la vaccinovigilance. On remercie également tous les membres présents ou passés du Groupe de travail sur la vaccinovigilance pour leur contribution au groupe de travail, et ce, tout particulièrement, Tania Diener, qui a agi à titre de première coprésidente provinciale. Enfin, il convient de souligner la contribution des nombreux membres de l'ASPC qui ont appuyé le groupe pendant toutes ces années, ainsi que les éléments du Comité canadien sur l'immunisation, pour leurs commentaires, leur rétroaction et leur expertise considérable au profit du Groupe de travail sur la vaccinovigilance. 


\section{Conflit d'intérêts}

Aucun

\section{Financement}

Le Groupe de travail sur la vaccinovigilance est financé par I'ASPC, ainsi que sous forme de soutien non financier des membres du groupe de travail faisant don de leur temps, et, à l'occasion, des coûts de déplacement afin de participer aux réunions.

\section{Références}

(1) Comité consultatif FPT sur la santé de la population et la sécurité de la santé. Stratégie nationale d'immunisation : Rapport final 2003. Rapport du Comité consultatif FPT sur la santé de la population et la sécurité de la santé (CCSPSS) à la Conférence des sous-ministres FPT de la santé. Ottawa : Agence de la santé publique du Canada; 2004.

http://www.phac-aspc.gc.ca/publicat/nis-sni-03/pdf/nat_imm_strat_f.pdf [consulté en octobre 2014].

(2) Oxford Dictionaries. Définition en anglais du mot vigilance. http://www.oxforddictionaries.com/definition/english/vigilance

(3) Council for International Organizations of Medical Sciences (CIOMS) and World Health Organization (WHO). Definition and Application of Terms for Vaccine Pharmacovigilance. Report of CIOMS/WHO Working Group on Vaccine Pharmacovigilance. Geneva: CIOMS and WHO; 2012. http://whqlibdoc.who.int/publications/2012/9789290360834_eng.pdf

(4) Agence de la santé publique du Canada. Déclaration de manifestations cliniques inhabituelles (MCl) à la suite d'une immunisation au Canada, Guide de l'utilisateur pour remplir et soumettre les rapports de déclaration de $\mathrm{MCl}$. [mis à jour le 14 juin 2014]. http://www.phac-aspc.gc.ca/im/aefi-essi_guide/index-fra.php

(5) Montalban, J.M., Ogbuneke, C., Hilderman, T. Analyse et renforcement du programme de sécurité des vaccins au Manitoba. Relevé des maladies transmissibles au Canada, vol. 40(S-3):S33-39, 4 décembre 2014.

(6) MacDonald, L., Naus, M. Les coulisses de la santé publique : Investigation des signes d'effets secondaires suivant l'immunisation (ESSI) en Colombie-Britannique. Relevé des maladies transmissibles au Canada, vol. 40(S-3):S24-32, 4 décembre 2014. 